\title{
Modelo matemático para populações de Aedes spp. com oviposição em ambientes aquáticos e secos
}

\author{
Alessandra A. C. Alves ${ }^{1}$ \\ José L. A. Fernandes ${ }^{2}$ \\ CEFET-MG, Belo Horizonte, MG \\ Lillia dos S. B. Silva ${ }^{3}$ \\ SEMED/Prefeitura Municipal de Sabará, Sabará, MG \\ Álvaro E. Eiras ${ }^{4}$ \\ UFMG, Belo horizonte, MG
}

\begin{abstract}
Resumo. O Aedes aegypti e o Aedes albopictus são os principais vetores transmissores de arboviroses no mundo como a dengue, zika, febre de chikungunya e a febre amarela, com maior incidência global desses vetores em regiões tropicais. A pluviosidade exerce considerável influência no desenvolvimento do Aedes spp., favorecendo o aumento do número de criadouros disponíveis para postura de ovos, ocasionando correlação positiva com a incidência de doenças. Um estudo teórico-numérico-computacional do modelo proposto, foi realizado com a finalidade de reproduzir a dependência dos parâmetros entomológicos com a pluviosidade acumulada semanalmente de uma determinada região, para investigar a relação entre a escolha do local de oviposição pela fêmea do mosquito, que pode ocorrer em ambientes secos ou aquáticos, e como essa escolha pode impactar na população de fêmeas. O sistema possui três equações diferenciais não lineares que representa a dinâmica das populações de ovos em ambientes secos e aquáticos e a população de fêmeas sob efeito de variáveis pluviométricas, sendo validado através da comparação com dados experimentais de capturas obtidos pelo monitoramento entomológico da cidade de Caratinga, Minas Gerais, Brasil. Verificou-se que o modelo descreve o ciclo de vida do mosquito, sendo capaz de auxiliar no controle e monitoramento do Aedes.
\end{abstract}

Palavras-chave. Aedes spp., Sistema Dinâmico, Oviposição, Pluviosidade

\section{Introdução}

A incidência de arboviroses como a dengue, zika e febre de chikungunya transmitidas pelo Aedes spp. aumentou drasticamente nas últimas décadas, sendo que $99 \%$ dessas doenças são causadas pelo Aedes aegypti e o Aedes albopictus, com maior incidência em áreas tropicais e subtropicais em todo o planeta segundo a OMS. As mudanças no clima, urbanização desordenada, o aumento de movimentações de pessoas e mercadorias e a falta de métodos eficazes de estratégias de controle são fatores que contribuem para a transmissão de doenças [14].

Estudos mostram que o ciclo de vida do Aedes spp. é influenciado pela temperatura, umidade [6] e chuvas [9], onde a fêmea adulta após o repasto sanguíneo segue em busca de um habitat adequado para efetuar a postura de ovos, que poderá ocorrer em ambientes secos ou aquáticos, com a possível eclosão dos ovos dependente de condições climáticas [5]. Em períodos chuvosos a fêmea

\footnotetext{
1 alessandraangelita@gmail.com.

2 acebal@cefetmg.br

${ }^{3}$ lilliabarsante@gmail.com.

4 alvaro@icb.ufmg.br.
} 
do mosquito encontra uma quantidade maior de água parada para depositar seus ovos, o ambiente urbano propicia o desenvolvimento do vetor com o aumento de criadouros artificiais, favorecendo a eclosão desses ovos livremente no verão [2]. As características dos locais podem desempenhar um importante papel no critério de escolha da oviposição. Em condições de temperaturas mais elevadas, acima de $35^{\circ} \mathrm{C}$, há uma diminuição na taxa de oviposição, enquanto que em condições de umidade mais alta há um aumento na postura de ovos e o comportamento dessa oviposição pode exercer influência na transmissão de doenças e pode ser significativo na criação de técnicas de vigilância e controle ( [3], [7]).

Modelos matemáticos relacionados a variáveis climáticas associadas ao ciclo de vida do mosquito, vem sendo desenvolvidos para auxiliar na prevenção e controle do vetor transmissor da doença, fornecendo uma aproximação simplificada a fim de descrever a dinâmica populacional do vetor em áreas urbanas, dentre esses trabalhos podemos citar ( [1], [11], [12], [13], [15]).

Neste trabalho, pesquisaremos em que medida a postura de ovos pela fêmea do mosquito em ambientes aquáticos e em ambientes secos, é capaz de modular a população alada e contribuir para descrever a sazonalidade do ciclo de vida do vetor, sendo que os ovos podem durar mais de um ano em condições climáticas desfavoráveis para o seu desenvolvimento entrando no processo de quiescência. A compreensão do processo de oviposição do Aedes spp. mais especificamente a compreensão da resistência à dessecação do ovo, pode fornecer informações sobre sua ecologia, dinâmica populacional, o momento adequado para efetuar mecanismos de controle além de considerar medidas de biossegurança [10]. Para isso, propomos e validamos um modelo matemático computacional que modela as populações de ovos e a população de fêmeas após o repasto sanguíneo com dependência pluviométrica.

\section{Modelagem}

Este modelo tem por objetivo descrever a dinâmica da oviposição da fêmea do Aedes spp., bem como o processo de escolha do local para efetuar a postura de ovos, como ambientes aquáticos e ambientes secos. Por simplicidade, a população aquática do mosquito foi reduzida apenas a população de ovos. O modelo é representado por um sistema dinâmico com três compartimentos e dependência pluviométrica:

$$
\begin{aligned}
\frac{d E_{W}}{d t} & =\phi_{W}(p(t))\left(1-\frac{E_{W}}{C}\right) F-\left(\mu_{E_{W}}(p(t))+\alpha(p(t))+\zeta_{E_{W}}(t)\right) E_{W} \\
\frac{d E_{D}}{d t} & =\phi_{D}(p(t))\left(1-\frac{E_{D}}{C}\right) F-\left(\mu_{E_{D}}(p(t))+\alpha(p(t))+\zeta_{E_{D}}(t)\right) E_{D} \\
\frac{d F}{d t} & =\alpha(p(t))\left(E_{W}+E_{D}\right)-\left(\mu_{F}(p(t))+\zeta_{F}(t)\right) F .
\end{aligned}
$$

A definição com sentido biológico para a região do espaço de estados do sistema dinâmico é:

$$
\Psi=\left\{\left(E_{W}, E_{D}, F\right) \in \mathbb{R}_{+}^{3} \mid E_{W} \geq 0 ; E_{D} \geq 0 ; F \geq 0 ; 0<E_{W}<C \text { e } 0<E_{D}<C\right\} .
$$

Com isso, as populações de fêmeas após o repasto sanguíneo são não negativas e as populações de ovos não ultrapassam a capacidade de suporte do ambiente.

A equação (1) descreve as populações de ovos em ambientes aquáticos $E_{W}(t)$, a equação (2) descreve as populações de ovos em ambientes secos $E_{D}(t)$ e a equação $(3)$ descreve a população de fêmeas após o repasto sanguíneo $F(t)$. Os parâmetros entomológicos relacionados às taxas de mortalidade natural per capita dos ovos nos ambientes aquoso e seco serão dadas respectivamente 
por $\mu_{E_{W}}$ e $\mu_{E_{D}}$. A taxa de mortalidade per capita dos mosquitos fêmeas dada por $\mu_{F}$. A taxa de oviposição em ambiente aquoso por unidade de fêmea é representada por $\phi_{W}\left(1-\frac{E_{W}}{C}\right)$, onde $\phi_{W}$ é a taxa de oviposição intrínseca em ambiente aquoso, e a taxa de oviposição em ambiente seco por unidade de fêmea é representada por $\phi_{D}\left(1-\frac{E_{D}}{C}\right)$, onde $\phi_{D}$ é a taxa de oviposição intrínseca em ambiente seco, $C$ é a capacidade de suporte do ambiente. As populações de ovos $E_{W}$ e $E_{D}$ desenvolvem-se a uma taxa per capita $\alpha$ e $\zeta_{E_{W}}, \zeta_{E_{D}}$ e $\zeta_{F}$ representam as taxas de mortalidade adicional per capita devido a adição de controle na respectiva população.

A taxa líquida de reprodução básica do modelo proposto neste trabalho é representada por $Q_{0}=Q_{W}+Q_{D}$, cuja equação é:

$$
Q_{0}=\frac{\phi_{W} \alpha}{\left(\mu_{F}+\zeta_{F}\right)\left(\alpha+\mu_{E_{W}}+\zeta_{E_{W}}\right)}+\frac{\phi_{D} \alpha}{\left(\mu_{F}+\zeta_{F}\right)\left(\alpha+\mu_{E_{D}}+\zeta_{E_{D}}\right)} .
$$

O modelo possui um ponto de equilíbrio trivial, que trata-se do equilíbrio onde não há populações de mosquitos

$$
P_{0}=\left(E_{W}^{*}, E_{D}^{*}, F^{*}\right)=(0,0,0),
$$

e dois pontos de equilíbrio não triviais, cujos pontos de equilíbrio em função de (4) são:

$$
P_{1}=\left(E_{W}^{* *}, E_{D}^{* *}, F^{* *}\right)
$$

em que

$$
\begin{aligned}
& E_{W}^{* *}=\frac{C}{2}\left(\frac{-\left(Q_{0}-2 Q_{W}+2 Q_{W}^{2}\right)+\sqrt{\left(Q_{0}-2 Q_{W}\right)^{2}+4 Q_{W}^{2}\left(Q_{0}-Q_{W}\right)^{2}}}{Q_{W}\left(Q_{0}-2 Q_{W}\right)}\right) \\
& E_{D}^{* *}=\left(\frac{1}{Q_{W}\left(1-\frac{E_{W}^{* *}}{C}\right)}-1\right) E_{W}^{* *} \quad \text { e } F^{* *}=\frac{\alpha\left(E_{W}^{* *}+E_{D}^{* *}\right)}{\left(\mu_{F}+\zeta_{F}\right)}
\end{aligned}
$$

e

$$
P_{2}=\left(E_{W}^{* * *}, E_{D}^{* * *}, F^{* * *}\right)
$$

em que

$$
\begin{aligned}
& E_{W}^{* * *}=\frac{C}{2}\left(\frac{-\left(Q_{0}-2 Q_{W}+2 Q_{W}^{2}\right)-\sqrt{\left(Q_{0}-2 Q_{W}\right)^{2}+4 Q_{W}^{2}\left(Q_{0}-Q_{W}\right)^{2}}}{Q_{W}\left(Q_{0}-2 Q_{W}\right)}\right) \\
& E_{D}^{* * *}=\left(\frac{1}{Q_{W}\left(1-\frac{E_{W}^{* * *}}{C}\right)}-1\right) E_{W}^{* * *} \text { e } F^{* * *}=\frac{\alpha\left(E_{W}^{* * *}+E_{D}^{* * *}\right)}{\left(\mu_{F}+\zeta_{F}\right)} .
\end{aligned}
$$

$\mathrm{O}$ ciclo de vida do Aedes spp. apresenta uma grande sensibilidade à presença de chuvas, produzindo consideravelmente muitos indivíduos. Contudo, para maiores volumes de chuvas, a população não conserva a mesma sensibilidade. Devido a isso, uma lei de potência [12] foi atribuída à dependência das taxas de oviposição, da taxa de desenvolvimento e das taxas de mortalidade do vetor com a chuva. Para efeito de simplificação, consideramos o mesmo valor $r$ para a potência dos parâmetros $\phi_{W}, \phi_{D}, \alpha, \mu_{E w}, \mu_{E d}$ e $\mu_{F}$. Este valor foi estimado pelo erro quadrático médio $s^{2}$ existente entre os dados simulados de fêmeas após o repasto sanguíneo e dados amostrais de Índice Médio de Fêmeas Aedes - IMFA concernente ao cenário de estudo da respectiva cidade. 


\section{Resultados}

As simulações do sistema dinâmico proposto neste trabalho foram obtidas numericamente pelo método de Runge-Kutta de quarta ordem no software MATLAB ${ }^{\circledR}$. O modelo sem intervenção de controle foi validado comparando os dados obtidos pela simulação computacional, com os dados reais de Índice Médio de Fêmeas Aedes - IMFA e de precipitação acumulada p (mm) por semana epidemiológica (SE), as amostras de dados utilizadas de precipitação acumulada foram relativas a SE $(9$ - 52) de 2009 a SE (1 - 52) de 2010 da cidade de Caratinga, Minas Gerais, Brasil, provenientes do Banco de Dados Meteorológicos para Ensino e Pesquisa - BDMEP, do Instituto Nacional de Meteorologia - INMET. Foram utilizadas como condição inicial as coordenadas do ponto de equilíbrio não trivial da primeira semana epidemiológica do cenário de pesquisa e não foram utilizadas ações de controle extra nas populações do vetor $\left(\zeta_{E_{W}}(t)=\zeta_{E_{D}}(t)=\zeta_{F}(t)=0\right)$. As faixas dos parâmetros utilizados no modelo estão representados na Tabela 1, o parâmetro $C$ foi considerado constante no decorrer de todo o cenário de estudo, estimando seu valor como $C=1$.

Tabela 1: Faixas dos parâmetros entomológicos do Aedes empregados nas equações do modelo proposto.

\begin{tabular}{cccc}
\hline Parâmetro & Faixa & Referência & Unidade \\
\hline$\phi_{W}$ & $0,5680-6,3616$ & Estimada & $\mathrm{dia}^{-1}$ \\
$\phi_{D}$ & $0,4320-4,8384$ & Estimada & $\mathrm{dia}^{-1}$ \\
$\mu_{F}$ & $0,033-0,046$ & Estimada & $\mathrm{dia}^{-1}$ \\
$\alpha$ & $0,05-0,19$ & Estimada & $\mathrm{dia}^{-1}$ \\
$\mu_{E_{W}}$ & $0,0053-0,0098$ & Estimada & $\mathrm{dia}^{-1}$ \\
$\mu_{E_{D}}$ & $0,0044-0,0161$ & Estimada & $\mathrm{dia}^{-1}$ \\
\hline
\end{tabular}

Os critérios de Routh-Hurwitz para o modelo, foram verificados computacionalmente no horizonte de estudo, resultando em (i) o ponto de equilíbrio não trivial $P_{1}$ será estável, uma vez que, todos os coeficientes $\left(a_{0}, a_{1}, a_{2}\right.$ e $\left.a_{3}\right)$ e o determinante $\Delta=a_{1} a_{2}-a_{3}$ possuem valores positivos; (ii) o ponto de equilíbrio trivial $P_{0}$ será instável, uma vez que, apenas os coeficientes $a_{0}$ e $a_{1}$ são positivos; e (iii) o ponto de equilíbrio não trivial $P_{2}$ será instável, uma vez que, o coeficiente $a_{2}$ e o determinante $\Delta=a_{1} a_{2}-a_{3}$ são negativos.

A Figura 1 mostra o progresso de $s^{2}$, o erro quadrático médio em função da potência $r$ do modelo proposto, com $r \in[0,1 \quad 1,0]$ e passo $h=0,01$. Nesse intervalo, os valores da potência $r$ que reduzem o $s^{2}$ estão relacionados as defasagens de duas e três semanas para a cidade de Caratinga. Para avaliar a dinâmica da população do vetor, foi considerada a potência $r$ relativa ao menor valor de $s^{2}$, correspondente a três semanas do cenário de estudo da cidade em questão. $\mathrm{O}$ valor do erro quadrático médio encontrado foi $s^{2}=1,94877.10^{-3}$ e o valor da potência $r=0,39$.

As Figuras 2, 3 e 4 mostram a evolução temporal da dinâmica evolutiva das populações de ovos em ambiente aquático $E_{W}(t)$, ambiente seco $E_{D}(t)$ e fêmeas após o repasto sanguíneo $F(t)$ onde os picos e vales das populações coincidem com os picos e vales de pluviosidade p (mm), mostrando a influência da chuva no ciclo evolutivo do Aedes spp. Em períodos de estiagem prolongada, as populações sofrem um declínio considerável, porém não chegam a extinguir-se, provavelmente isso deve-se a resistência a dessecação dos ovos e a existência de potenciais criadouros artificiais do mosquito nesse intervalo de tempo. O caso da evolução das populações de ovos estarem equiparadas, pode estar ligado ao fato de termos considerado por simplicidade o mesmo valor para a taxa de desenvolvimento do vetor da fase aquática para a alada. 


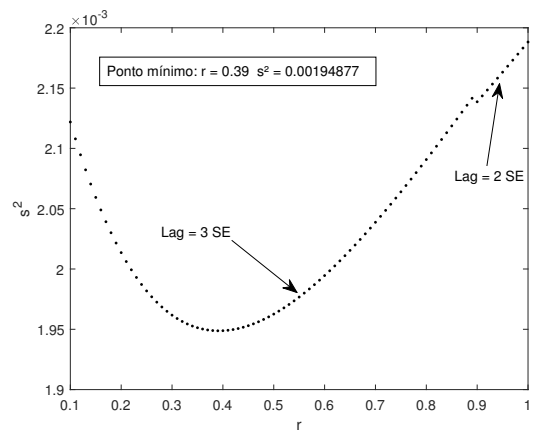

Figura 1: Dependência do erro quadrático médio $s^{2}$ e da defasagem (Lag) $d$, com a potência $r$ do modelo proposto para a cidade de Caratinga, Minas Gerais, Brasil.

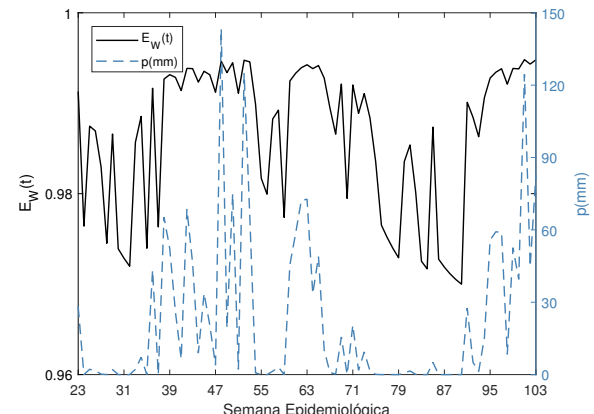

Figura 2: Curva da evolução da população de ovos em ambiente aquático $E_{W}(t)$ de acordo com a pluviosidade $\mathrm{p}(\mathrm{mm})$.

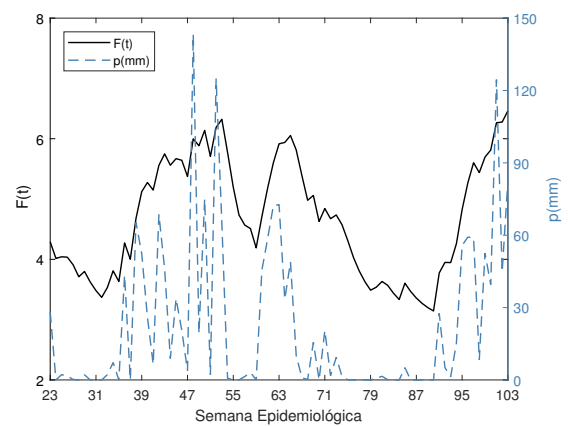

Figura 4: Curva da evolução da população de mosquitos fêmeas $F(t)$ após o repasto sanguíneo de acordo com a pluviosidade $\mathrm{p}$ $(\mathrm{mm})$.

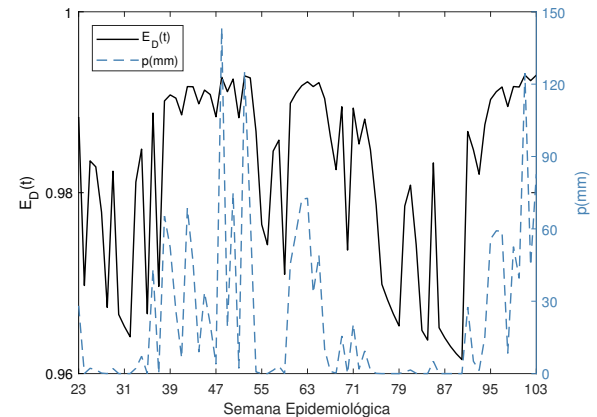

Figura 3: Curva da evolução da população de ovos em ambiente seco $E_{D}(t)$ de acordo com a pluviosidade $\mathrm{p}(\mathrm{mm})$.

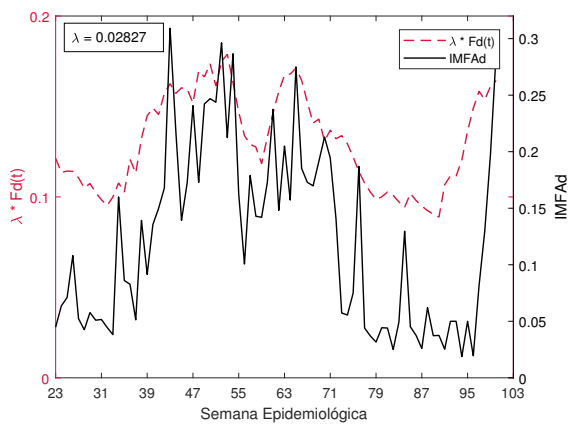

Figura 5: Dados amostrais de Índice Médio de Fêmeas Aedes, com defasagem calculada a partir da correlação cruzada e do fator escala $\lambda$.

A Figura 5 mostra a comparação qualitativa entre os dados simulados de fêmeas após o repasto sanguíneo do modelo proposto e os dados amostrais de IMFA, com a defasagem calculada a partir da correlação cruzada entre os dados e do fator escala $\lambda=0,02827$, que é um fator positivo que ajusta verticalmente a escala relativa dos dados simulados de fêmeas após o repasto sanguíneo com 
dados amostrais de IMFA após a correlação cruzada. A evolução da população de fêmeas após o repasto sanguíneo do modelo seguiu a tendência de evolução de IMFA com um atraso médio entre os picos e os vales de três semanas epidemiológicas para a cidade de Caratinga com o valor máximo da correlação equivalente a $M=0,73783$. De acordo com a classificação de [4], temos que a intensidade $M$ das correlações entre duas sequências foi forte para a cidade de Caratinga.

Para fins de comparação, iremos contrapor o resultado alcançado relacionado ao menor valor do erro quadrático médio $s^{2}$ do modelo proposto neste trabalho com os trabalhos de [1] e [11]. Foram realizadas simulações numéricas sem ação de controle adicional nas populações dos respectivos modelos entomológicos, utilizando dados amostrais de IMFA e de precipitação acumulada p (mm) por semana epidemiológica (SE) no cenário de pesquisa na cidade de Caratinga. A Tabela 2 mostra a comparação entre o valor do erro quadrático médio $s^{2}$ e da defasagem $d$ obtidos entre os dados de IMFA e os dados simulados de fêmeas após o repasto sanguíneo do modelo em estudo e dos modelos matemáticos entomológicos: [1] e (1), (15) e (29) do trabalho de [11]. O menor valor do $s^{2}$ foi obtido na simulação do modelo (29) de [11] com um atraso médio entre as curvas de três semanas e o pior valor foi obtido na simulação do modelo de [1] com um atraso médio entre as curvas de uma semana.

Tabela 2: Comparação do erro quadrático médio $s^{2}$ e da defasagem $d$ obtidos a partir dos dados amostrais de Índice Médio de Fêmeas Aedes e os dados simulados de fêmeas após o repasto sanguíneo dos modelos [1] e (1), (15) e (29) do trabalho de [11] para a cidade de Caratinga, Minas Gerais, Brasil.

\begin{tabular}{ccc}
\hline Modelo & Valor de $s^{2}$ & Valor de $d$ \\
\hline (Modelo proposto) & $1,94877 \cdot 10^{-3}$ & $3 \mathrm{SE}$ \\
\hline$(1)$ de $[11]$ & $1,20324 \cdot 10^{-3}$ & $4 \mathrm{SE}$ \\
\hline$(15)$ de $[11]$ & $1,40502 \cdot 10^{-3}$ & $3 \mathrm{SE}$ \\
\hline$(29)$ de $[11]$ & $1,16499 \cdot 10^{-3}$ & $3 \mathrm{SE}$ \\
\hline$[1]$ & $3,39340 \cdot 10^{-3}$ & $1 \mathrm{SE}$ \\
\hline
\end{tabular}

\section{Conclusões}

Neste trabalho, mostramos um estudo teórico-numérico-computacional de um modelo matemático compartimental não linear, que descreve a dependência dos parâmetros entomológicos relacionados ao ciclo de vida do Aedes spp. em relação ao processo de escolha do ambiente de oviposição com a dependência pluviométrica acumulada semanalmente de uma determinada região. A implementação do modelo proposto e seus resultados, foram comparados com dados amostrais de IMFA da cidade de Caratinga, Minas Gerais, Brasil, correspondendo as expectativas de que o modelo tem um comportamento semelhante ao esperado para a evolução no desenvolvimento do vetor. Com isso o modelo poderá especificar a população, contribuindo para relatar as flutuações sazonais no ciclo de vida do mosquito. Como trabalhos futuros, acrescentaremos nos parâmetros do modelo proposto a influência da temperatura, além de ações de controle do Aedes spp. e o refinamento das faixas dos parâmetros entomológicos.

\section{Referências}

[1] Barsante, L. D. S. Dependência entre pluviosidade e população de fêmeas Aedes aegypti grávidas descritas através de um sistema dinâmico não linear, Dissertação de Mestrado, CEFET-MG, 2012. 
[2] Corrêa, F. V. e Palhares, J. M. Aumento de casos de dengue relacionados com fatores climáticos e o meio socioambiental no município de Oiapoque-AP-Brasil: período de 2008 a 2013, Ciência Geográfica, 20(1):58-70, 2016.

[3] Costa, E. A. P. D. A., Santos, E. M. D. M., Correia, J. C. and Albuquerque, C. M. R. D. Impact of small variations in temperature and humidity on the reproductive activity and survival of Aedes aegypti (Diptera, Culicidae), Revista Brasileira de Entomologia, SciELO Brasil, 54(3):488-493, 2010. DOI: 10.1590/S0085-56262010000300021.

[4] Dancey, C. P. and Reidy, J. Statistics without maths for psychology, 7th Edition. Pearson education, New York, 2017.

[5] Mullen, G. R. and Durden, L. A. Medical and veterinary entomology, 3rd Edition. Academic press, 2019.

[6] Reinhold, J. M., Lazzari, C. R. and Lahondère, C. Effects of the Environmental Temperature on Aedes aegypti and Aedes albopictus Mosquitoes: A review, Insects, 9(4):158, 2018. DOI: 10.3390/insects9040158.

[7] Rey, J. R. and O'Connell, S. M.Oviposition by Aedes aegypti and Aedes albopictus: Influence of congeners and of oviposition site characteristics, Journal of Vector Ecology, Wiley Online Library, 39(1):190-196, 2014.

[8] Rodrigues, H. S., Monteiro, M. T. T. and Torres, D. F. M. Sensitivity analysis in a dengue epidemiological model, Conference papers in science, volume 2013, Hindawi, 2013. DOI: $10.1155 / 2013 / 721406$.

[9] Santos, I. C. D. S., Braga, C., De Souza, W. V., De Oliveira, A. L. S. and Regis, L. N. The influence of meteorological variables on the oviposition dynamics of Aedes aegypti (Diptera: Culicidae) in four environmentally distinct areas in northeast Brazil, Memórias do Instituto Oswaldo Cruz, volume 115, 2020. DOI: 10.1590/0074-02760200046.

[10] Silva, H. H. G. D. e Silva, I. G. D. Influência do período de quiescência dos ovos sobre o ciclo de vida de Aedes aegypti (Linnaeus, 1762)(Diptera, Culicidae) em condições de laboratório, Revista da Sociedade Brasileira de Medicina Tropical, 32(4):349-355, 1999. DOI: 10.1590/S0037-86821999000400003.

[11] Silva, L. D. S. B. Proposição e análise de modelos determinísticos com parâmetros meteorológicos para descrição da população de mosquitos Aedes Spp. e otimização da eficácia do controle entomológico. Tese de Doutorado, CEFET-MG, 2018.

[12] Silva, L. D. S. B., Vasconcelos, A. S. V., Sanches, A. L., Cardoso, R. T. N., Fernandes, J. L. A. e Eiras, A. E. Otimização mono-objetivo no controle do mosquito Aedes aegypti por meio de um modelo de duas populações com influência da precipitação, TEMA (São Carlos), SciELO Brasil, 20(1):197-216, 2019. DOI: 10.5540/tema.2019.020.01.0197.

[13] Valdez, L. D., Sibona, G. J., and Condat, C. A. Impact of rainfall on Aedes aegypti populations, Ecological Modelling, Elsevier, 385:96-105, 2018. DOI: 10.1016/j.ecolmodel.2018.07.003.

[14] WHO \& IAEA. Guidance framework for testing the sterile insect technique as a vector control tool against Aedes-borne diseases, Geneva: World Health Organization and the International Atomic Energy Agency, 2020. Licence: CC BY-NC SA 3.0 IGO.

[15] Yang, H. M. Assessing the influence of quiescence eggs on the dynamics of mosquito Aedes aegypti, Applied Mathematics, Scientific Research Publishing 5(17):2696-2711, 2014. DOI: 10.4236/am.2014.517257. 